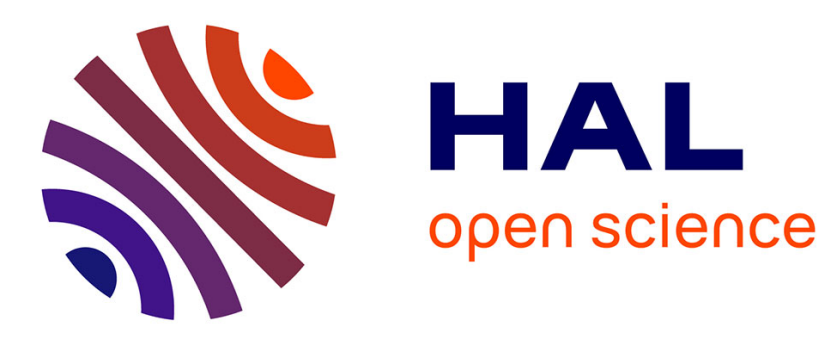

\title{
State estimation of an octorotor with unknown inputs. Application to radar imaging
}

Thomas Chevet, Maria Makarov, Cristina Stoica Maniu, Israel Hinostroza, Pierre Tarascon

\section{- To cite this version:}

Thomas Chevet, Maria Makarov, Cristina Stoica Maniu, Israel Hinostroza, Pierre Tarascon. State estimation of an octorotor with unknown inputs. Application to radar imaging. 21st International Conference on System Theory, Control and Computing, Oct 2017, Sinaia, Romania. pp.723-728, 10.1109/ICSTCC.2017.8107122 . hal-01630173

\section{HAL Id: hal-01630173 \\ https://hal.science/hal-01630173}

Submitted on 7 May 2018

HAL is a multi-disciplinary open access archive for the deposit and dissemination of scientific research documents, whether they are published or not. The documents may come from teaching and research institutions in France or abroad, or from public or private research centers.
L'archive ouverte pluridisciplinaire HAL, est destinée au dépôt et à la diffusion de documents scientifiques de niveau recherche, publiés ou non, émanant des établissements d'enseignement et de recherche français ou étrangers, des laboratoires publics ou privés. 


\title{
State Estimation of an Octorotor with Unknown Inputs. Application to Radar Imaging
}

\author{
Thomas Chevet*, Maria Makarov ${ }^{\dagger}$, Cristina Stoica Maniu ${ }^{\dagger}$, Israel Hinostroza ${ }^{\ddagger}$, Pierre Tarascon* \\ ${ }^{*}$ Department of Automatic Control \\ CentraleSupélec \\ France, Gif-sur-Yvette, F91192 \\ E-mail: thomas.chevet@supelec.fr; \\ pierre.tarascon@supelec.fr \\ ${ }^{\dagger}$ Laboratoire des Signaux et Systèmes \\ CentraleSupélec-CNRS-Univ. Paris-Sud, \\ Université Paris-Saclay \\ France, Gif-sur-Yvette, F91192 \\ E-mail: maria.makarov@ centralesupelec.fr; \\ cristina.stoica@supelec.fr \\ ${ }_{\text {SONDRA Lab }}$ \\ CentraleSupélec \\ France, Gif-sur-Yvette, F91192 \\ E-mail: israel.hinostroza@supelec.fr
}

\begin{abstract}
This paper focuses on the design of a linear Kalman filter and an extended Kalman filter for the estimation of an octorotor unmanned aerial vehicle's (UAV) state in the context of Synthetic Aperture Radar image reconstruction. A comparison to a linear interpolation method is also proposed. The Kalman filters are developed based on a complete nonlinear model of the UAV and its linearized form. A particularity of the considered platform is that the control signals are not measured and have to be estimated as well as the UAV's state. The proposed techniques are then tested on a UAV simulator and a radar imaging simulator.

Keywords-UAV, State Estimation, Kalman Filter, Extended Kalman Filter, Synthetic Aperture Radar
\end{abstract}

\section{INTRODUCTION}

Since they are lighter, cheaper and easier to deploy than the traditional radar platforms such as planes and satellites [1], unmanned aerial vehicles (UAV) [2] are a new center of interest for radar applications. To this aim, several studies have been done to show their usefulness, e.g. [3], [4]. In this context, this paper is part of ongoing research and of an educational project, its objective being to design an UAV with an embedded radar system for a wide scope of civilian applications like crop field monitoring, rescue operations after avalanches, damage evaluation after natural disasters, etc. In order to reconstruct radar images from the acquired data, the knowledge of the UAV's position and attitude has to be known as accurately as possible.

For modeling and testing purposes, a commercially available octorotor (the ARF-MikroKopter Okto-XL, Mikrokopter, HySystems GMBH) which is compatible with the required payload and provides redundancy with its eight rotors is considered in this paper. Several control schemes for attitude control or trajectory tracking have been presented on Mikrokopter platforms [5] and on the Okto-XL [6], [7]. A large spectrum of works concerning the usage of Kalman filters to estimate attitude and trajectory of a UAV has been published, such as the state estimation of winged UAVs [8], [9], [10] or the attitude estimation of multirotor UAVs [6], [11]. All these papers focus on the use of extended or unscented Kalman filters with measurements provided by inertial measurement units and GPS knowing the control signals.
In the context of radar applications employing the octorotor ARF-MikroKopter Okto-XL, using the model proposed in [7], this paper focuses in a first stage on the design of a linear Kalman filter and an extended Kalman filter. In a second stage, their comparison with a linear interpolation technique for the state estimation of an octorotor UAV is considered in view of radar applications, more precisely for radar image reconstruction. The control signal on this platform is not available for measurements. It is then considered to be a perturbation and the UAV's state vector has to be augmented to include it. The measurements are provided by an inertial measurement unit and an embedded GPS with an important noise level. Due to these particularities, a linear interpolation method is not always efficient enough and Kalman filtering techniques are tested to improve the quality of radar image reconstruction. A flight with the UAV is simulated as well as a radar acquisition. The flight data are processed through the Kalman filters. The filtered data is then processed through a radar image reconstruction algorithm to compare the efficiency of the different estimation methods.

Section II presents the principles of Synthetic Aperture Radar, the sensors presented on the platform, the model used to design the Kalman filters, the particularities of the considered UAV and the model's sampling. Section III is dedicated to the classical and extended Kalman algorithms and their assumptions. Simulation conditions and results of the state estimation and radar image reconstruction are detailed in Section IV. Finally, concluding remarks are drawn in Section V.

\section{SYSTEM DESCRIPTION AND ESTIMATION SPECIFICATIONS}

\section{A. Synthetic Aperture Radar}

Synthetic Aperture Radar (SAR) image formation is based on the coherent sum of the scattered waves due to the periodic (Period Repetition Frequency - PRF) illumination of a scene by the radar [12], ideally at constant speed, hence the illumination is uniformly distributed in space. For stripmap mode, sideway illumination (relative to the UAV's movement) of the scene with constant inclination, altitude, speed and linear 
TABLE I: Requirements for radar applications

\begin{tabular}{lll}
\hline Radar & Platform speed & $2.5 \mathrm{~m} / \mathrm{s}$ \\
specifications & Ground resolution & $0.8 \times 0.8 \mathrm{~m}$ \\
& Height of flight & $10 \mathrm{~m}$ \\
& Ground range & $2 \mathrm{~m} \mathrm{to} 40 \mathrm{~m}$ \\
\hline Trajectory & Translation speed & $2.5 \pm 0.2 \mathrm{~m} / \mathrm{s}$ \\
specifications & Maximum trajectory deviation & $<0.5 \mathrm{~m}$ \\
& Operational altitude & $10 \mathrm{~m}$ \\
& Stabilized attitude & \\
\hline
\end{tabular}

trajectory, is desired. For small UAVs, such as the one used in this paper, this is rarely the case. The radar specifications for the $\mathrm{X}$ band FM Continuous Wave (FMCW) Synthetic Aperture Radar to be used are summarized in Table I. Deviations from the ideal known linear trajectory induce changes in the expected phase history of the scatters (objects in the scene), producing errors in the synthesis of the SAR image. Errors of the same kind are produced when estimating the position of the radar. These errors can be compensated with signal processing autofocus techniques, in combination with the information of the sensors of the UAV, up to a certain degree, see [12, Chapter 5.2] for more details. In order to comply with the radar specifications, by limiting the phase history errors due to trajectory deviations, the desired control performances of the UAV's movement are specified in Table I.

\section{B. Dynamical model of the UAV}

1) Presentation of the platform: The considered octorotor (Fig. 1 [7]) is the ARF-MikroKopter OktoXL. The drone main parameters are provided in Table II. This drone is equipped with a set of sensors comprising an inertial measurement unit (IMU), an altimeter, a magnetometer and a GPS. The UAV's microcontroller provides fused and filtered data on the position of the drone (denoted by $X, Y$ and $Z$ ), its attitude $(\varphi, \theta$ and $\psi)$ and its horizontal speed $\left(V_{h}\right)$ at a low sampling rate. This filtering process eliminates the biases and provides noisy measurements. In order to compare the reconstruction algorithms used to obtain exploitable data for radar applications at a high sample rate (with sampling time $\left.T_{e}\right)$ two extreme cases will be distinguished:

- Case 1 considers a quite high noise level on the position provided by the GPS, corresponding to the precision of the sensors as if they were directly acquired by the user;

- Case 2 considers a lower noise level on the position, corresponding to the quantization noise of the signals provided by the microcontroller.

The noise standard deviation values are presented in Table III. The measurement signals are then retrieved by a computer via the microcontroller's serial port during the UAV's flight.

2) Dynamical model: The nonlinear equations of motion in the state-space form are written using the following state vector: $\mathbf{x}=$ $\left(\begin{array}{llllllllllll}X & Y & Z & \varphi & \theta & \psi & V_{x} & V_{y} & V_{z} & \omega_{x} & \omega_{y} & \omega_{z}\end{array}\right)^{T}$ where $X, Y$ and $Z$ are the position of the drone in the Earth's frame, $V_{x}, V_{y}$ and $V_{z}$ their time derivatives, $\varphi, \theta$ and $\psi$ the Euler angles defining the orientation of the drone in
TABLE II: Drone parameters: notations and values

\begin{tabular}{ll}
\hline Arm lengths (long, short) & $L=0.455 \mathrm{~m}, l=0.349 \mathrm{~m}$ \\
Total mass with one battery & $m=2.56 \mathrm{~kg}$ \\
Inertia components & $I_{x x}=0.0869, I_{y y}=0.0873$, \\
& $I_{z z}=0.1683 \mathrm{~kg} \cdot \mathrm{m}^{2}$ \\
Air drag coefficient & $d_{w}=0.03 \mathrm{~kg} \cdot \mathrm{m}^{-1}$ \\
\hline
\end{tabular}

TABLE III: Measurement noise standard deviation

\begin{tabular}{ll}
\hline Case 1 & $\sigma_{X, Y, Z}=50 \mathrm{~cm}$ \\
Case 2 & $\sigma_{X, Y, Z}=10 \mathrm{~cm}$ \\
Both cases & $\sigma_{\varphi, \theta, \psi}=0.07 \mathrm{rad}, \sigma_{V_{h}}=7 \mathrm{~cm} / \mathrm{s}$ \\
\hline
\end{tabular}

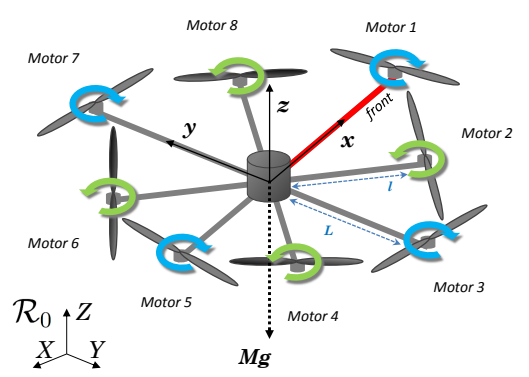

Fig. 1: Considered octorotor and reference frames.

the Earth's frame, and $\omega_{x}, \omega_{y}$ and $\omega_{z}$ the angular speeds of the drone expressed in its own frame.

The complete nonlinear model describing the dynamics of the drone is further used:

$$
\begin{aligned}
\dot{X}= & V_{x}, \quad \dot{Y}=V_{y}, \quad \dot{Z}=V_{z} \\
\dot{\varphi}= & \omega_{x}+\mathrm{s} \varphi \frac{\mathrm{s} \theta}{\mathrm{c} \theta} \omega_{y}+\mathrm{c} \varphi \frac{\mathrm{s} \theta}{\mathrm{c} \theta} \omega_{z} \\
\dot{\theta}= & \mathrm{c} \varphi \omega_{y}-\mathrm{s} \varphi \omega_{z} \\
\dot{\psi}= & \frac{\mathrm{s} \varphi}{\mathrm{c} \theta} \omega_{y}+\frac{\mathrm{c} \varphi}{\mathrm{c} \theta} \omega_{z} \\
m \dot{V}_{x}= & (c \psi c \theta) F_{x}^{R}+(\mathrm{c} \psi \mathrm{s} \theta \mathrm{s} \varphi-\mathrm{s} \psi \mathrm{c} \varphi) F_{y}^{R} \\
& +(\mathrm{c} \psi \mathrm{s} \theta \mathrm{c} \varphi+\mathrm{s} \psi \mathrm{s} \varphi) F_{z}^{R}+F_{x}^{a i r} \\
m \dot{V}_{y}= & (\mathrm{s} \psi \mathrm{c} \theta) F_{x}^{R}+(\mathrm{s} \psi \mathrm{s} \theta \mathrm{s} \varphi+\mathrm{c} \psi \mathrm{c} \varphi) F_{y}^{R} \\
& +(\mathrm{s} \psi \mathrm{s} \theta \mathrm{c} \varphi-\mathrm{c} \psi \mathrm{s} \varphi) F_{z}^{R}+F_{y}^{a i r} \\
m \dot{V}_{z}= & (-\mathrm{s} \theta) F_{x}^{R}+(\mathrm{c} \theta \mathrm{s} \varphi) F_{y}^{R}+(\mathrm{c} \theta \mathrm{c} \varphi) F_{z}^{R} \\
& +F_{z}^{a i r}-m g \\
I_{x x} \dot{\omega}_{x}= & \left(I_{y y}-I_{z z}\right) \omega_{y} \omega_{z}+\tau_{x}^{R} \\
I_{y y} \dot{\omega}_{y}= & \left(I_{z z}-I_{x x}\right) \omega_{x} \omega_{z}+\tau_{y}^{R} \\
I_{z z} \dot{\omega}_{z}= & \left(I_{x x}-I_{y y}\right) \omega_{x} \omega_{y}+\tau_{z}^{R}
\end{aligned}
$$

The numerical values of the parameters are provided in Table II. Here, $I_{x x}, I_{y y}$ and $I_{z z}$ are the components of the UAV's inertia, $F_{x}^{R}, F_{y}^{R}$ and $F_{z}^{R}$ are the components of the resulting propeller's force and $\tau_{x}^{R}, \tau_{y}^{R}$ and $\tau_{z}^{R}$ are the components of the resulting propeller's torque (the last six being expressed in the drone's frame, being denoted by the superscript $R$ ). The functions $\sin (\cdot)$ and $\cos (\cdot)$ are respectively represented by $\mathrm{s} \cdot$ and $\mathrm{c} \cdot$. The air drag effects are assumed to be: $\mathbf{F}^{\text {air }}=-d_{w}\left\|\mathbf{V}_{\text {rel }}\right\| \mathbf{V}_{\text {rel }}$ where $\mathbf{V}_{\text {rel }}=\mathbf{V}-\mathbf{V}^{w}$ 
is the relative speed between the UAV (its speed being $\mathbf{V}$ ) and the wind (its speed being $\mathbf{V}^{w}$ ) and $d_{w}$ the air drag coefficient.

3) Particularities of the platform: The resulting propeller force and torque $\mathbf{F}^{R}$ and $\tau^{R}$ are functions of the squared propellers' speeds $\Omega_{i}, i=1, \ldots, 8$. These rotational speeds are the model's inputs. However, in the case of our platform, these input signals are not available and the control law is not precisely known and not considered in the estimation model. As no input are acquired, it will be considered as a perturbation in the following. Thus, the perturbation vector used is $\mathbf{P}=\left(\begin{array}{lllllllll}F_{x}^{R} & F_{y}^{R} & F_{z}^{R} & \tau_{x}^{R} & \tau_{y}^{R} & \tau_{z}^{R} & V_{x}^{w} & V_{y}^{w} & V_{z}^{w}\end{array}\right)^{T}$ where $V_{x}^{w}, V_{y}^{w}$ and $V_{z}^{w}$ are the component of the wind's speed along the three axes of the Earth's frame. For estimation purposes, the perturbation is supposed to be constant over the time, i.e. $\dot{\mathbf{P}}=\mathbf{0}_{9 \times 1}$.

Moreover, the sensors have noise levels presented in Table III. Hence the acquired signals are noisy and inaccurate. In addition, because of the way the measurements are acquired (as presented in Paragraph II-B1) the acquiring rate of the measurement is not steady and varies between 0.4 and $0.6 \mathrm{~s}$. The acquired signals are the linear position $X, Y$ and $Z$, the angular position $\varphi, \theta$ and $\psi$ and the UAV's horizontal speed

$$
V_{h}=\sqrt{V_{x}^{2}+V_{y}^{2}} \text {. }
$$

In order to reconstruct radar images, the ideal situation would be to have distance variation between two successive points (after estimation) under the radar's wavelength $(0.03 \mathrm{~m})$.

\section{Sampling of the dynamical model}

In order to estimate the perturbation vector $\mathbf{P}$ as well as the state vector $\mathbf{x}$, an extended state vector is defined as $\mathbf{x}_{e}=$ $\left(\begin{array}{ll}\mathbf{x}^{T} & \mathbf{P}^{T}\end{array}\right)^{T}$. The continuous time state-space model of the $\mathrm{UAV}$ is:

$$
\dot{\mathbf{x}}_{e}(t)=\boldsymbol{f}_{\boldsymbol{c}}\left(\mathbf{x}_{e}(t)\right)+\mathbf{v}_{\mathbf{c}}(t)
$$

where $f_{c}$ is a $21 \times 1$ vector function grouping the equations (1) to (10), the last nine lines being zeros (according to the constant model of $\mathbf{P}$ ) and $\mathbf{v}_{\mathbf{c}}$ is a white noise quantifying the modeling errors.

This model is sampled with the $T_{e}$ sampling time:

$$
\left\{\begin{aligned}
\mathbf{x}_{e}[k+1]= & \mathbf{x}_{e}[k]+T_{e} \boldsymbol{f}_{\boldsymbol{c}}\left(\mathbf{x}_{e}[k]\right)+\mathbf{v}[k] \\
\mathbf{z}[k] & =\boldsymbol{h}\left(\mathbf{x}_{e}[k]\right)+\mathbf{w}[k]
\end{aligned}\right.
$$

where the function $\cdot+T_{e} \boldsymbol{f}_{\boldsymbol{c}}(\cdot)$ will be called $\boldsymbol{f}$ to simplify the notations. The measurement function is $\boldsymbol{h}=$ $\left(\begin{array}{lllllll}X & Y & Z & \varphi & \theta & \psi & V_{h}\end{array}\right)^{T}$ and $\mathbf{z}$ is the system's output vector. Here, $\mathbf{v}$ (process noise) and $\mathbf{w}$ (measurement noise) are uncorrelated white noises. Since the measurement is already a discrete process on the UAV, w's variance is not affected by the sampling process. The process noise $\mathbf{v}$ being a tuning parameter for the estimation, quantifying the uncertainties in the modeling and sampling processes, its variance is not affected by the sampling process.

Then, because the state-space model is nonlinear, it is linearized in order to further use a linear Kalman filter. At the hovering state, the velocities, both linear and angular, are equal to zero. In order to simplify the linearization problem ${ }^{1}$, the air drag force is considered to have three components $F_{x}^{a i r}$, $F_{y}^{a i r}$ and $F_{z}^{a i r}$ that will replace the last three components of $\mathbf{P}$. When the model is linearized, it is sampled as:

$$
\left\{\begin{aligned}
\mathbf{x}_{e}[k+1] & =\boldsymbol{F} \mathbf{x}_{e}[k] \\
\mathbf{z}[k] & =\boldsymbol{H} \mathbf{x}_{e}[k]
\end{aligned} \quad \text { with } \boldsymbol{F}=\mathrm{e}^{T_{e} \boldsymbol{J}}\right.
$$

where $\boldsymbol{J}$ and $\boldsymbol{H}$ are respectively the Jacobians of $\boldsymbol{f}_{\boldsymbol{c}}$ and $\boldsymbol{h}$ evaluated at the considered equilibrium point.

A problem will appear when computing the Jacobian of $\boldsymbol{h}$ because the derivative of its last component $V_{h}$ (see (11)) ends up being divided by zero. Hence, this last component of $\boldsymbol{h}$ will not be considered in the linearized model.

\section{KALMAN FILTERING}

The Kalman filtering method is well known and has been abundantly used in aircraft's state estimation [13], [14]. In order to reconstruct radar images, the UAV's position has to be estimated from the measurements. The objective is to compare the efficiency of Kalman filtering over a simple linear interpolation of the measurements. Hence, a linear Kalman filter is first developed, then an extended Kalman filter is proposed.

\section{A. Linear Kalman filtering}

A linear discrete-time state-space model of the considered system is needed to use a linear Kalman filter:

$$
\left\{\begin{aligned}
\mathbf{x}_{e}[k+1] & =\boldsymbol{F} \mathbf{x}_{e}[k]+\mathbf{v}[k] \\
\mathbf{z}[k] & =\boldsymbol{H} \mathbf{x}_{e}[k]+\mathbf{w}[k]
\end{aligned}\right.
$$

where $\mathbf{v}[k]$ and $\mathbf{w}[k]$ are zero-mean white noises (the process and measurement noise), with $\boldsymbol{E}\left(\mathbf{w}[k] \mathbf{w}[j]^{T}\right)=\boldsymbol{R} \delta_{k j}$, $\boldsymbol{E}\left(\mathbf{v}[k] \mathbf{v}[j]^{T}\right)=\boldsymbol{Q} \delta_{k j}$ and $\boldsymbol{E}(\mathbf{w}[k] \mathbf{v}[j])=\mathbf{0}$, where $\delta_{k j}$ is the Kronecker delta function and $\boldsymbol{E}(\cdot)$ is the mathematical expectation of $\cdot$.

In the first stage of the estimation process, the filter predicts the value at sample $k+1$ of the extended state vector $\mathbf{x}_{e}[k+1 \mid k]$ and its associated prediction error covariance matrix $\boldsymbol{P}[k+1 \mid k]$ using the estimate $\widehat{\mathbf{x}}_{e}[k \mid k]$ at sample $k$ of the state vector and the estimated prediction error covariance matrix $\boldsymbol{P}[k \mid k]$ :

$$
\left\{\begin{aligned}
\widehat{\mathbf{x}}_{e}[k+1 \mid k] & =\boldsymbol{F} \widehat{\mathbf{x}}_{e}[k \mid k] \\
\boldsymbol{P}[k+1 \mid k] & =\boldsymbol{F P}[k \mid k] \boldsymbol{F}^{T}+\boldsymbol{Q}
\end{aligned}\right.
$$

If no measurement is acquired at the sample $k+1$, only the prediction stage is performed. However, if a measurement is acquired, the prediction obtained in the first stage is corrected using the measurement model:

$$
\left\{\begin{aligned}
\widehat{\mathbf{x}}_{e}[k+1 \mid k+1] & =\widehat{\mathbf{x}}_{e}[k+1 \mid k]+\boldsymbol{K}_{k+1} \mathbf{e}[k+1] \\
\boldsymbol{P}[k+1 \mid k+1] & =\left(\boldsymbol{I}-\boldsymbol{K}_{k+1} \boldsymbol{H}\right) \boldsymbol{P}[k+1 \mid k] \\
\boldsymbol{K}_{k+1} & =\boldsymbol{P}[k+1 \mid k] \boldsymbol{H}^{T} \boldsymbol{V}_{k+1}^{-1} \\
\boldsymbol{V}_{k+1} & =\boldsymbol{H P}[k+1 \mid k] \boldsymbol{H}^{T}+\boldsymbol{R} \\
\mathbf{e}[k+1] & =\mathbf{z}[k+1]-\boldsymbol{H} \widehat{\mathbf{x}}_{e}[k+1 \mid k]
\end{aligned}\right.
$$

where $\boldsymbol{K}_{k+1}$ is the Kalman gain at step $k+1$ and $\mathbf{e}$ is the innovation sequence.

\footnotetext{
${ }^{1}$ Linearization details on the UAV dynamic model can be found in [7].
} 


\section{B. Extended Kalman filtering}

A nonlinear discrete-time state-space model of the considered system is needed to use an extended Kalman filter:

$$
\left\{\begin{aligned}
\mathbf{x}_{e}[k+1]= & \boldsymbol{f}\left(\mathbf{x}_{e}[k]\right)+\mathbf{v}[k] \\
\mathbf{z}[k] & =\boldsymbol{h}\left(\mathbf{x}_{e}[k]\right)+\mathbf{w}[k]
\end{aligned}\right.
$$

where $\mathbf{v}$ and $\mathbf{w}$ have the same properties as with the classical Kalman filter.

The two stages of the estimation process are globally the same as with the classical Kalman filter with $\boldsymbol{F}_{k}$ and $\boldsymbol{H}_{k}$ being the Jacobians of $\boldsymbol{f}$ and $\boldsymbol{h}$ evaluated at each iteration:

$$
\boldsymbol{F}_{k}=\boldsymbol{I}_{21}+\left.T_{e} \frac{\partial \boldsymbol{f}_{c}}{\partial \mathbf{x}_{e}}\right|_{\widehat{\mathbf{x}}_{e}[k \mid k]} \text { and } \boldsymbol{H}_{k}=\left.\frac{\partial \boldsymbol{h}}{\partial \mathbf{x}_{e}}\right|_{\widehat{\mathbf{x}}_{e}[k \mid k-1]}
$$

Moreover, the first equation of (16) becomes:

$$
\widehat{\mathbf{x}}_{e}[k+1 \mid k]=\boldsymbol{f}\left(\widehat{\mathbf{x}}_{e}[k \mid k]\right)
$$

and the innovation defined in the last equation of (17) is:

$$
\mathbf{e}[k+1]=\mathbf{z}[k+1]-\boldsymbol{h}\left(\widehat{\mathbf{x}}_{e}[k+1 \mid k]\right)
$$

\section{Simulation AND RESUlTs}

\section{A. SAR simulator and image formation algorithm}

The UAV's state was simulated with a Matlab/Simulink simulator implementing the nonlinear model described in Section II. A LQI (Linear Quadratic Integral) controller was used based on a LQ (Linear Quadratic) controller proposed for this UAV in [7].

The reference trajectory corresponds first to a take-off until an altitude of $10 \mathrm{~m}$, followed by a translation on the $\mathrm{X}$-axis at a constant speed of $2.5 \mathrm{~m} / \mathrm{s}$. In order to test the robustness of the estimation to the presence of wind disturbances, wind gusts (of $20 \mathrm{~s}$ each) of $10 \mathrm{~km} / \mathrm{h}$ in the $-\mathrm{X}$ direction (at $t=25$ $\mathrm{s}$ and $t=120 \mathrm{~s}$ ) and $20 \mathrm{~km} / \mathrm{h}$ in the $-\mathrm{Y}$ direction (at $t=55 \mathrm{~s}$ and at $t=145 \mathrm{~s}$ ) are applied. Once this trajectory is simulated, a Gaussian zero-mean white noise is added to the measured states with the standard deviations defined in Table III.

The measurements are then processed through the Kalman filters. The measurement noise variance matrix $\boldsymbol{R}$ is filled with the variances obtained from the standard deviations in Table III. The process noise variance matrix $\boldsymbol{Q}$ is a tuning parameter. Its value was set using a particle swarm optimization (PSO) algorithm [15], [16], [17]. The particles are vectors of size 22, the 21 first coordinates being the components of a diagonal matrix $\mathfrak{Q}$ and the last one being a scaling parameter factor $q$ such that $\boldsymbol{Q}=q \mathfrak{Q}$. The $q$ parameter is introduced to avoid numerical errors both in the PSO algorithm and in the filter algorithm. The PSO algorithm minimizes the quadratic error between the estimated and the simulated trajectory defined above. The estimated trajectory is obtained with an extended Kalman filter in the case 1 designed with the considered UAV model (13).

Finally, the simulated linear position and the estimated linear position are processed through a SAR simulator. Two targets, or scatter, are placed along the trajectory to be observed by the UAV. Radar phase history simulations are carried out using the parameters in Table IV considering a FMCW radar. For the image formation, back projection algorithm is used for its simplicity [18]. Additionally, it is naturally adapted when the illumination of the scene is not uniform in space (for example variations on the UAV speed during radar acquisition with constant PRF).

\section{B. Simulation results}

The acquired measurements are processed through a linear and an extended Kalman filter based on the models presented in Section II-C. The filter is initialised with $\widehat{\mathbf{x}}_{e}[0 \mid 0]=\mathbf{0}$ and $\boldsymbol{P}[0 \mid 0]=\boldsymbol{I}$, except for the linear velocities $V_{x}, V_{y}$ and $V_{z}$ being initialized to $0.1 \mathrm{~m} / \mathrm{s}$ in the case of the extended Kalman filter to avoid singularity.

The noise level is reduced with both filters (Fig. 2 and Fig. 3, red dash-dotted lines) but the extended Kalman filter is more efficient since it carries more information at each step and the model used is more accurate. On Fig. 4a, the error on the trajectory is lower after the Kalman filtering compared to the error after the linear interpolation. However, the extended Kalman filter takes more iterations to converge (between 6 and $10 \mathrm{~s}$ ) than the linear Kalman filter (less than $1 \mathrm{~s}$ ). This way, if an extended filter is used, the sensor acquisition has to start before the radar acquisition to allow the filter convergence.

The efficiency of the Kalman filtering in case 1 is shown on Fig. 5 since the images obtained with the filtered data (Fig. 5d and Fig. 5c) are better focused than the one obtained with the interpolated data (Fig. 5b). For example, the extended Kalman filter is more efficient since the position of Scatter 2 is correctly formed (Fig. 5c). It can be seen that, with the linear interpolation (Fig. 5b), two scatters' appear at the same range instead of only one for both Scatter 1 and Scatter 2.

Since the noise level is quite high in case 1 (low quality sensors were considered) and the control signal is unknown, the noise level on the filtered data is still quite high (the error is around $20 \mathrm{~cm}$ ) after filtering. This remaining error on the trajectory can be derived from Fig. 4a. Moreover, the UAV's position is highly dependent on its attitude as seen on (1) to (10). Thus, because of the high noise level on the angle measurements and their sparsity (implying sparse updates of the control signal which is estimated as well) the estimated position undergoes variations regardless of the filter when it is supposed to remain constant.

Finally, the Kalman filtering approach offers good results when the noise level is of the magnitude considered in case 1. If the measurement signal is already filtered and the noise level

TABLE IV: Main SAR parameters for simulation

\begin{tabular}{ll}
\hline Wavelength & $0.03 \mathrm{~m}$ \\
Tx bandwidth & $200 \mathrm{MHz}$ \\
Azimuth beamwidth (aperture antenna) & 24 \\
PRF & $2 \mathrm{kHz}$ \\
Scatter 1 position & Azimuth $=91.04 \mathrm{~m}$ \\
& Ground range $=30 \mathrm{~m}$ \\
Scatter 2 position & Azimuth $=96.04 \mathrm{~m}$ \\
& Ground range $=20 \mathrm{~m}$ \\
\hline
\end{tabular}



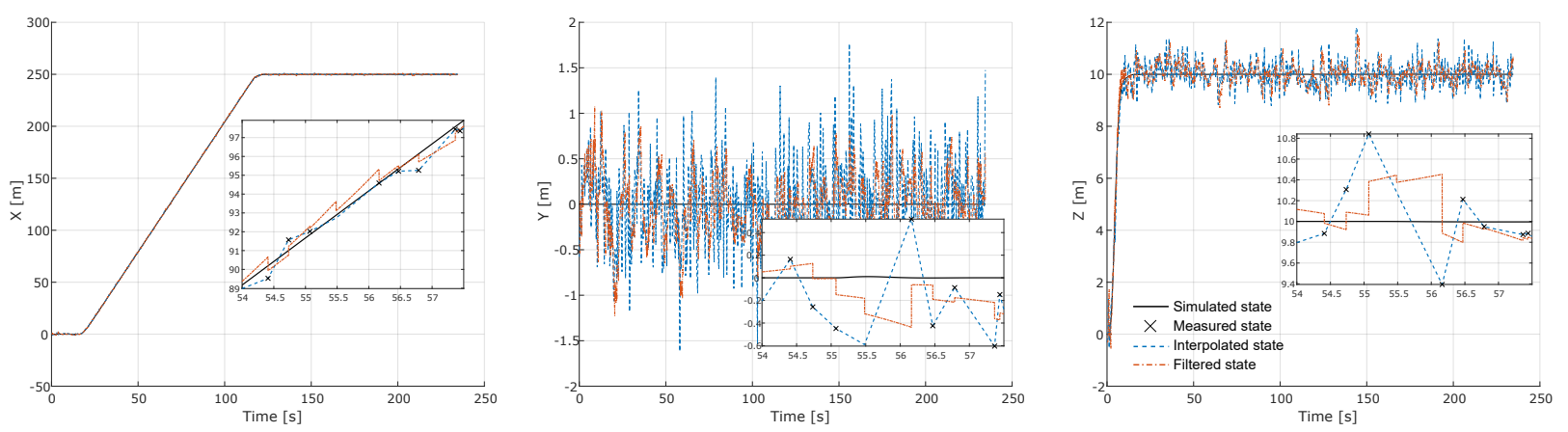

Fig. 2: Estimation of the $X$ (left), $Y$ (center) and $Z$ (right) states with a linear Kalman filter.
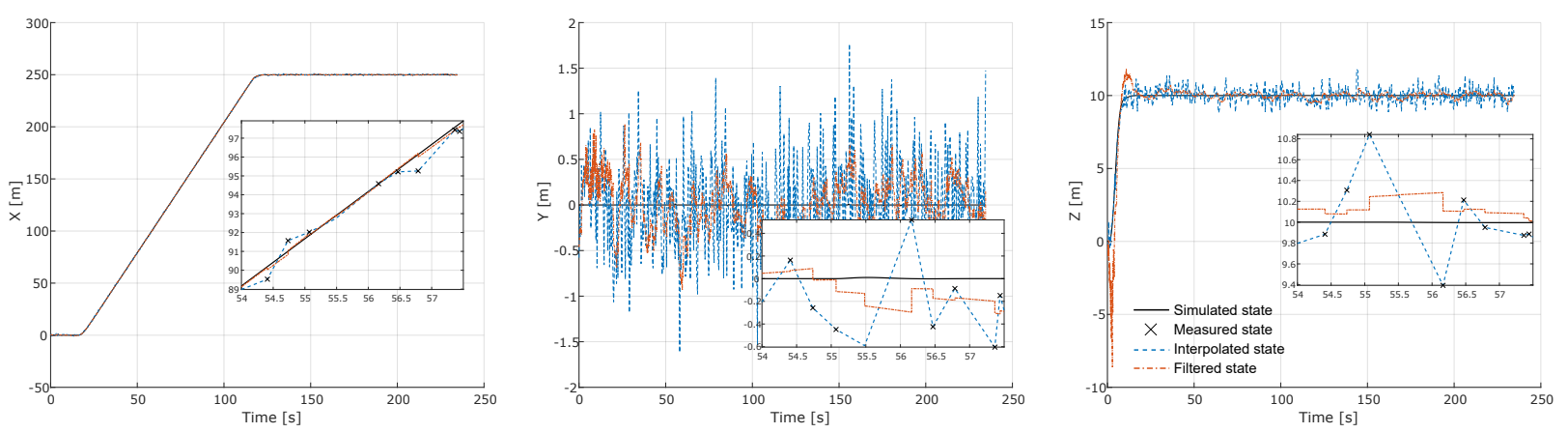

Fig. 3: Estimation of the $X$ (left), $Y$ (center) and $Z$ (right) states with an extended Kalman filter.
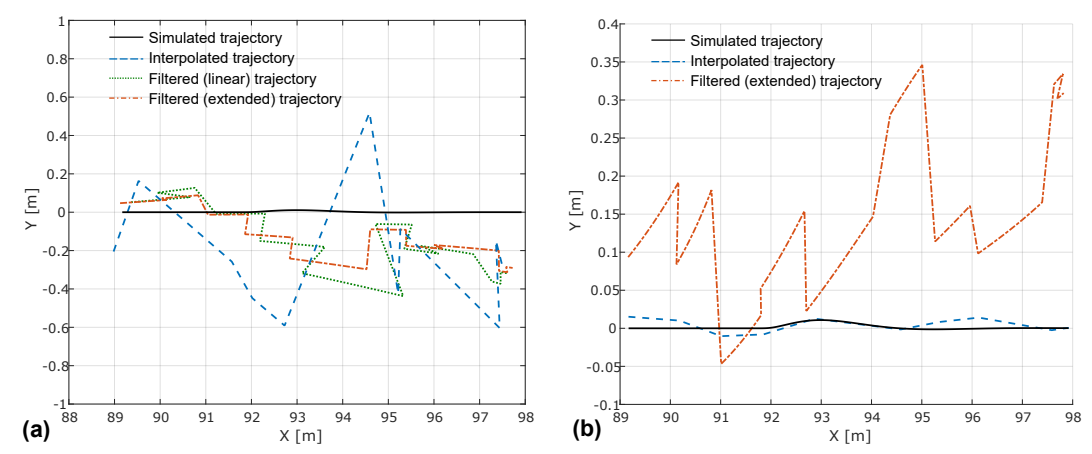

Fig. 4: Trajectory in the $X Y$ plane in case 1 (a) and in case 2 (b).

is of the magnitude considered in case 2, the Kalman filtering approach (or at least with a classical or an extended Kalman filter) is less efficient than the interpolation approach. In case 2 , the SAR image obtained with the filtered measurement (Fig. 5f) is more blurred than the image obtained with the interpolated data (Fig. 5e). Indeed, the standard deviation on the linear position was $\approx 10 \mathrm{~cm}$ and, as shown in Fig. $4 \mathrm{~b}$, the error on the trajectory is bigger after the Kalman filtering than after the linear interpolation. Thus, for a low noise level as in case 2, a simple linear interpolation of the measurement can be enough.

\section{CONCLUSION}

A linear and an extended Kalman filter have been designed to estimate the state of an octorotor drone. The drone's control signals were unknown and the sensors were of low quality, producing unsteady measurements with a high noise level. The estimation error is smaller with the extended Kalman filter (EKF) than with the linear Kalman filter since the model used is more complete with the EKF. The efficiency of this extended Kalman filter over a linear interpolation or a linear Kalman filtering method is demonstrated by producing Synthetic Aperture Radar (SAR) images with a simulator. The image obtained with the extended filter is clearer than the other two SAR images. When the noise level is not as high as considered when simulating the measurements, the linear interpolation method can be more efficient than the Kalman filters. Finally, if the noise level is too high, neither the linear interpolation nor the Kalman filters are efficient for 

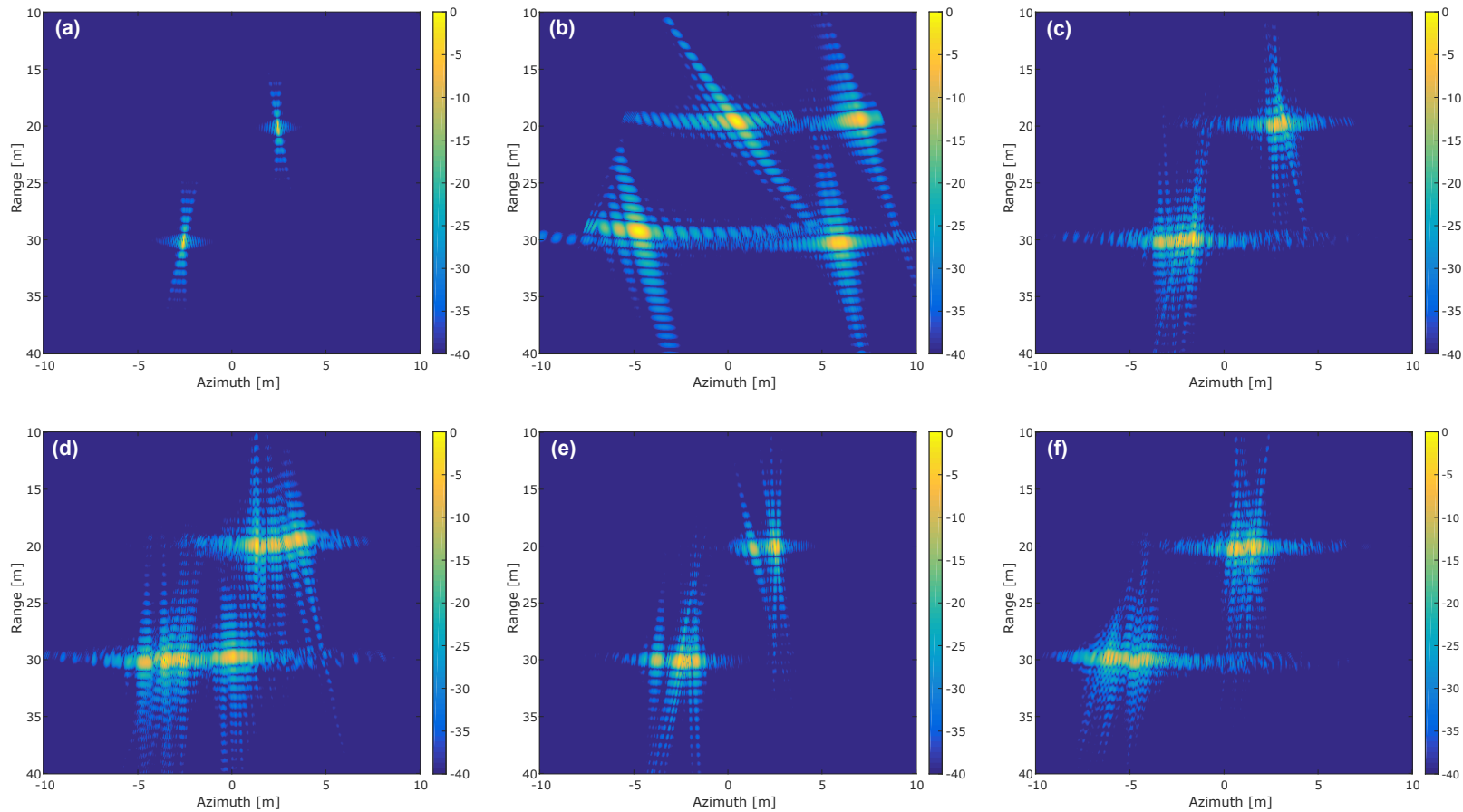

Fig. 5: SAR images obtained with the simulated state (a), the interpolated state in case 1 (b) and case 2 (e), the filtered (linear) state in case 1 (d) and the filtered (extended) state in case 1 (c) and case 2 (f).

\section{SAR image reconstruction.}

In future work, more advanced estimation methods will be investigated. For example, a two-stage Kalman filter will be used to perform a separate estimation of the perturbation vector and divide the problem's size. Moreover, an unscented Kalman filter would increase the accuracy of the estimation since no first order approximation is performed. In both cases, a more complex model of the perturbation vector will be tested. Finally, this work will be used to design an observer to use a Linear Quadratic Integral controller designed for octorotor drones.

\section{ACKNOWLEDGMENT}

The first author would like to thank prof. Dominique Beauvois for his teaching on the Kalman filtering techniques and the motivating interesting discussions on this subject.

\section{REFERENCES}

[1] A. Moreira, P. Prats-Iraola, M. Younis, G. Krieger, I. Hajnesk, and K. P. Papathanassiou, "A tutorial on synthetic aperture radar," IEEE Geoscience and remote sensing magazine, vol. 1, no. 1, 2013.

[2] R. Lozano, Unmanned aerial vehicles: Embedded control. John Wiley \& Sons, 2013.

[3] E. Zaugg, D. Hudson, and D. Long, "The BYU SAR: A small, studentbuilt SAR for UAV operation," in IEEE International Geoscience and Remote Sensing Symposium, 2006.

[4] J.-T. Gonzalez-Partida, P. Almorox-Gonzalez, M. Burgos-Garcia, and B.P. Dorta-Naranjo, "SAR system for UAV operation with motion error compensation beyond the resolution cell," Sensors, vol. 8, no. 5, 2008.

[5] I. Sa and P. Corke, "System identification, estimation and control for a cost effective open-source quadcopter," in IEEE International Conference on Robotics and Automation (ICRA), 2012.
[6] K. Bergman and J. Ekström, "Modeling, estimation and attitude control of an octorotor using PID and L1 adaptative control techniques," 2014 , Master Thesis.

[7] M. Makarov, C. Stoica Maniu, S. Tebbani, I. Hinostroza, M. Moreira Beltrami, J. Kienitz, R. Menegazzi, C. Sallé Moreno, T. Rocheron, and J. Rojas Lombarte, "Octorotor UAVs for radar applications: modeling and analysis for control design," in Workshop on Research, Education and Development of Unmanned Aerial Systems, Cancun, Mexico, 2015.

[8] B. O. Teixeira, L. A. Tôrres, P. Iscold, and L. A. Aguirre, "Flight path reconstruction-A comparison of nonlinear Kalman filter and smoother algorithms," Aerospace Science and Technology, vol. 15, no. 1, 2011.

[9] H. G. de Marina, F. J. Pereda, J. M. Giron-Sierra, and F. Espinosa, "UAV attitude estimation using unscented Kalman filter and TRIAD," IEEE Transactions on Industrial Electronics, vol. 59, no. 11, 2012.

[10] B. Kada, K. Munawar, M. Shaikh, M. Hussaini, and U. Al-Saggaf, "UAV attitude estimation using nonlinear filtering and low-cost mems sensors," IFAC-PapersOnLine, vol. 49, no. 21, 2016

[11] K. D. Sebesta and N. Boizot, "A real-time adaptive high-gain EKF, applied to a quadcopter inertial navigation system," IEEE Transactions on Industrial Electronics, vol. 61, no. 1, 2014.

[12] W. Carrara, R. Goodman, and R. Majewski, Spotlight Synthetic Aperture Radar: signal processing algorithms, ser. Artech House remote sensing library. Artech House, 1995.

[13] F. Auger, M. Hilairet, J. M. Guerrero, E. Monmasson, T. OrlowskaKowalska, and S. Katsura, "Industrial applications of the Kalman filter: A review," IEEE Transactions on Industrial Electronics, vol. 60, no. 12 2013.

[14] M. S. Grewal and A. P. Andrews, "Applications of Kalman filtering in aerospace 1960 to the present [historical perspectives]," IEEE Control Systems, vol. 30, no. 3, 2010.

[15] G. Sandou, "Particle swarm optimization: An efficient tool for the design of automatic control laws," in European Control Conference (ECC). IEEE, 2009.

[16] R. Poli, J. Kennedy, and T. Blackwell, "Particle swarm optimization," Swarm Intelligence, vol. 1, no. 1, 2007.

[17] M. Clerc, Particle Swarm Optimization, ser. ISTE. Wiley, 2010.

[18] M. Soumekh, Synthetic Aperture Radar Signal Processing. Wiley, 1999. 